\title{
DISTANCE MAGIC GRAPHS - A SURVEY
}

\author{
S. Arumugam ${ }^{1,2}$, Dalibor FronceK ${ }^{3}$ And N. Kamatchi ${ }^{4}$
}

${ }^{1}$ National Centre for Advanced Research

in Discrete Mathematics (n-CARDMATH), Kalasalingam University, Anand Nagar, Krishnankoil-626 190, India, s.arumugam.klu@gmail.com ${ }^{2}$ School of Electrical Engineering and Computer Science, The University of Newcastle, NSW 2308, Australia

${ }^{3}$ Department of Mathematics and Statistics, University of Minnesota Duluth, 1117 University Drive, Duluth, MN 55812-3000, U.S.A.

${ }^{4}$ National Centre for Advanced Research

in Discrete Mathematics ( $n$-CARDMATH), Kalasalingam University,

Anand Nagar, Krishnankoil-626 190, India, n_kamatchi@yahoo.com

\begin{abstract}
Let $G=(V, E)$ be a graph of order $n$. A bijection $f: V \rightarrow\{1,2, \ldots, n\}$ is called a distance magic labeling of $G$ if there exists a positive integer $k$ such that $\sum_{u \in N(v)} f(u)=k$ for all $v \in V$, where $N(v)$ is the open neighborhood of $v$. The $u \in N(v)$

constant $k$ is called the magic constant of the labeling $f$. Any graph which admits a distance magic labeling is called a distance magic graph. In this paper we present a survey of existing results on distance magic graphs along with our recent results, open problems and conjectures.

Key words: Distance magic labeling, magic constant, fair incomplete tournament.
\end{abstract}

2000 Mathematics Subject Classification: 05C78.

Received: 09-08-2011, revised: 09-09-2011, accepted: 04-12-2012. 